\title{
Vulvovaginal Candidiasis in Patients presenting with Vaginal Discharge in a Sri Lankan Setting
}

\author{
${ }^{1}$ Manjula Manoji Weerasekera, ${ }^{2}$ TD Chinthika P Gunasekara, ${ }^{3}$ Neluka Fernando, ${ }^{4}$ Janani Kottachchi \\ ${ }^{5}$ Tishani Methsela Wijesuriya, ${ }^{6}$ Achala Bogahawatta, ${ }^{7}$ Ramya Pathiraja
}

\begin{abstract}
Background: Vulvovaginal candidiasis (VVC) is a common infection among women. Due to the emerging role of nonalbicans Candida in VVC, it is important to periodically investigate the proportions of infection caused by different Candida species and antifungal resistance patterns.
\end{abstract}

Aims: This study aims to determine the frequency of occurrence of $\mathrm{VVC}$ in women presenting with vaginal discharge, possible risk factors associated with the disease, and antifungal susceptibility pattern of the isolates of Candida.

Materials and methods: High vaginal swabs from 158 women presenting with vaginal discharge at the gynecology clinic of Colombo South Teaching Hospital were studied. Ethical approval was granted from the Ethical Review Committee of the University of Sri Jayewardenepura (No.: 722/13). After species identification, antifungal susceptibility tests were performed using a standard disk diffusion technique. Probable risk factors for the infection were assessed using an intervieweradministered questionnaire. Statistical analysis was performed with Statistical Package for the Social Sciences (SPSS), using chi-square tests. All inferential statistics were tested at $p<0.05$.

Results: Candida albicans was isolated in 34 patients (22\%), and Candida famata was isolated from one patient. All direct microscopy-positive specimens were also culture positive. All the isolates were susceptible to antifungals tested. Among the study population, oral contraceptives (OCP) were used in $11 \%$ $(n=17)$ of patients $(p=0.001)$. Fourteen $(40 \%)$ of 35 Candida positive patients had diabetes mellitus $(p<0.05)$.

Conclusion: Candida albicans was the commonest species isolated from VVC in a Sri Lankan setting. The infection can safely be treated with commonly used antifungals. Diabetes mellitus and use of OCP are associated risk factors.

Keywords: Antifungals, Candida, Sri Lanka, Vulvovaginal candidiasis.

\footnotetext{
1,2,4,7 Senior Lecturer, ${ }^{3}$ Professor, ${ }^{5}$ Graduate ${ }^{6}$ Technical Officer

1-6Department of Microbiology, Faculty of Medical Sciences University of Sri Jayewardenepura, Nugegoda, Gangodawila Sri Lanka

${ }^{7}$ Department of Obstetrics and Gynecology, Faculty of Medical Sciences, University of Sri Jayewardenepura, Nugegoda Gangodawila, Sri Lanka

Corresponding Author: Manjula Manoji Weerasekera, Senior Lecturer, Department of Microbiology, Faculty of Medical Sciences, University of Sri Jayewardenepura, Nugegoda Gangodawila, Sri Lanka, Phone: +94714432870 , e-mail: mmweera@yahoo.com; mmweera@sjp.ac.lk
}

How to cite this article: Weerasekera MM, Gunasekara TDCP, Fernando N, Kottachchi J, Wijesuriya TM, Bogahawatta A, Pathiraja R. Vulvovaginal Candidiasis in Patients presenting with Vaginal Discharge in a Sri Lankan Setting. J South Asian Feder Obst Gynae 2016;8(2):123-126.

Source of support: Nil

Conflict of interest: None

Date of received: 8 Feb 2016

Date of acceptance: 25 Mar 2016

Date of publication: April 2016

\section{INTRODUCTION}

Vulvovaginal candidiasis (VVC) is experienced by $75 \%$ of women during their lifetime. ${ }^{1}$ The symptoms of VVC can impact the quality of life of women, leading to depression and sexual problems. Due to the significant involvement of non-albicans Candida species ${ }^{2,3}$ and their resistance to azoles, treatment of VVC is often difficult. ${ }^{4,5}$ The fungal species causing VVC and their antifungal susceptibility (AFS) patterns may vary with the geographical location and host factors. ${ }^{6}$ Hence, it is imperative to periodically investigate risk factors for VVC, the prevailing isolates, and their AFS patterns, to enable proper management and prevention of the condition.

\section{MATERIALS AND METHODS}

This study was a cross-sectional, prospective study conducted in 2012-2013 at the Obstetrics and Gynecology Professorial Unit, Colombo South Teaching Hospital, and Department of Microbiology, Faculty of Medical Sciences, University of Sri Jayewardenepura, Sri Lanka.

Ethical approval was granted by the Ethical Review Committee of the University of Sri Jayewardenepura, Sri Lanka (ethical approval number 722/13). One hundred and fifty-eight patients (between 20 and 80 years of age) with vaginal discharge were included in the study.

After a probable clinical diagnosis was made by the gynecologist, two high vaginal swabs were taken by a medical officer for laboratory identification. An interviewer-based questionnaire was filled by a researcher. The swabs were transported immediately to the laboratory of microbiology. 
A Gram stain was done on one swab and the other swab was used for culture isolation. Culture was done on two separate culture plates. The culture plates Sabouraud dextrose agar with or without cycloheximide $(100 \mu \mathrm{g} / \mathrm{mL})$ and chloramphenicol $(50 \mu \mathrm{g} / \mathrm{mL})$ were incubated at $37^{\circ} \mathrm{C}$ for 24 hours. Isolates were deemed significant if they were isolated on both culture plates. Isolated Candida species were identified up to species level by germ tube test, carbon assimilation, and sugar fermentation test.

Antifungal susceptibility testing was done on all the Candida strains that were isolated from cultures. MuellerHinton agar (MHA) supplemented with $2 \%$ glucose and $0.5 \mu \mathrm{g} / \mathrm{mL}$ methylene blue dye medium was used. A suspension of overnight cultures of $C$. albicans and C. famata was prepared in sterile saline and standardized to $0.5 \mathrm{McF}$ arland.

A sterile cotton swab was dipped into the suspension and rotated several times. The excess fluid from the swab was removed by pressing firmly against the inside wall above the fluid level. The dried surface of the MHA was inoculated by streaking the cotton swab according to the standard method to get an even distribution of the inoculum. Commercially prepared paper disks clotrimazole $(10 \mu \mathrm{g})$, itraconazole $(10 \mu \mathrm{g})$, ketoconazole $(10 \mu \mathrm{g})$, fluconazole $(25 \mu \mathrm{g})$, and miconazole ( $30 \mu \mathrm{g})$ were dispensed on each plate, according to a standard method described by the Clinical laboratories Standards Institute. ${ }^{7}$ The plates were incubated at $37^{\circ} \mathrm{C}$ for 24 hours, and then zone diameters were measured manually after 24 hours and interpreted as shown in Table 1. If no visible growth was shown, the plates were re-incubated for up to 24 hours more.

\section{RESULTS}

Among the 158 patients (between 20 and 80 years) with vaginal discharge, Candida species were isolated from $22 \%(n=35)$ patients. Of these, 34 isolates were $C$. albicans and only one patient had a non-albicans species. None of the patients were infected with multiple Candida species. Candida was isolated from 17 patients aged between 20 and 40 years ( $48.5 \%), 15$ patients aged between 41 and 60 years $(43 \%)$, and 3 patients over the age of 61 years $(0.1 \%)$.

Among the 35 culture positives, $51(n=18)$ and 26\% patients claimed that vaginal discharge and symptoms

Table 1: Zone diameter ranges

\begin{tabular}{lllll}
\hline & Potency & \multicolumn{3}{c}{ Zone of diameter $(\mathrm{mm})$} \\
\cline { 3 - 5 } Antifungal disk & $(\mu \mathrm{g})$ & Sensitive & Intermediate & Resistance \\
\hline Clotrimazole & 10 & $\geq 20$ & $12-19$ & $\leq 11$ \\
Itraconazole & 10 & $\geq 23$ & $14-22$ & $\leq 13$ \\
Ketoconazole & 10 & $\geq 30$ & $23-29$ & $\leq 22$ \\
Fluconazole & 25 & $\geq 19$ & $15-18$ & $\leq 14$ \\
Miconazole & 30 & $\geq 20$ & $12-19$ & $\leq 11$ \\
\hline
\end{tabular}

occurred post menstruation and during menstruation respectively. A total of $23 \%$ patients claimed that there was no relationship between vaginal discharge and menstruation.

The following symptoms were seen in the 35 culturepositive patients: Itching $(n=15,43 \%)$, odor $(n=14,40 \%)$, viscous discharge $(n=25,71 \%)$, watery discharge $(n=10$, $28 \%)$, white discharge $(\mathrm{n}=26,74 \%)$, and yellow discharge $(n=8,22 \%)$. Of these, the presence of white and viscous discharge were statistically significant $(p<0.05)$ than other types of discharges. Among the 35 culture positives, only two patients had used antibiotics during the past 2 months.

All the isolated strains of Candida species were susceptible to the tested antifungals: Fluconazole, clotrimazole, itraconazole, ketoconazole, and miconazole, depending on the measurement in their zone of inhibition (Table 2).

When referring to the whole population of patients $(n=158)$, diabetes mellitus had a significant association with candidal vaginal infections $(p=0.01)$. Oral contraceptives were used by 17 patients with vaginal discharge; seven of these patients had Candida infections, which was statistically significant $(p=0.01)$. Among the 26 patients who were in the menopausal stage, only five were confirmed to have Candida infections.

Seven patients were using contraceptive injections and only one patient among them was positive for Candida species $(p=0.61)$. Also $18 \%(n=28)$ of the patients were using the intrauterine contraceptive devices, and among them seven were confirmed to have Candida infection $(p=0.8)$. With regard to undergarments, those wearing nylon underwear $[n=21(23 \%)]$ showed culture positive with Candida. When inquired if the patients had visited a doctor earlier regarding the vaginal discharge, 38/158 gave a positive response. Interestingly, of these 38 patients, 34 had no improvement in symptoms despite the treatment. However, only 11 of these patients were positive for Candida by culture in the present study.

\section{DISCUSSION}

In the present study, VVC was seen in a lower percentage (22\%) of females when compared to a study in 1994 (32.4\%). In another study done in 2010, among patients attending the sexually transmitted infections (STI) clinic, $8 \%$ cases were reported. ${ }^{8}$ The lower percentage of the latter study may be due to a smaller sample size $(n=96)$ and the study being limited to a specific population (STI patients).

The low prevalence rate of VVC in our study was possibly due to improved knowledge and hygiene, as compared to the result reported a decade ago among 
Table 2: Results of the antifungal susceptibility testing

\begin{tabular}{|c|c|c|c|c|c|}
\hline \multirow[b]{2}{*}{ No. of culture positives } & \multicolumn{5}{|c|}{ Zone of inhibition $(\mathrm{mm})$} \\
\hline & Clotrimazole & Itraconazole & Ketoconazole & Fluconazole & Miconazole \\
\hline 1 & 31 & 20 & 35 & 36 & 27 \\
\hline 2 & 36 & 26 & 32 & 34 & 32 \\
\hline 3 & 34 & 32 & 38 & 40 & 32 \\
\hline 4 & 28 & 18 & 33 & 27 & 38 \\
\hline 5 & 29 & 20 & 31 & 32 & 25 \\
\hline 6 & 28 & 19 & 30 & 35 & 23 \\
\hline 7 & 31 & 21 & 35 & 34 & 27 \\
\hline 8 & 28 & 20 & 31 & 34 & 25 \\
\hline 9 & 34 & 22 & 37 & 35 & 26 \\
\hline 10 & 32 & 21 & 30 & 32 & 26 \\
\hline 11 & 30 & 21 & 35 & 33 & 26 \\
\hline 12 & 32 & 23 & 33 & 33 & 25 \\
\hline 13 & 31 & 22 & 37 & 33 & 29 \\
\hline 14 & 25 & 19 & 30 & 28 & 28 \\
\hline 15 & 28 & 21 & 30 & 32 & 22 \\
\hline 16 & 31 & 22 & 34 & 35 & 26 \\
\hline 17 & 30 & 20 & 32 & 32 & 22 \\
\hline 18 & 30 & 19 & 31 & 30 & 21 \\
\hline 19 & 28 & 20 & 30 & 32 & 25 \\
\hline 20 & 28 & 20 & 33 & 32 & 25 \\
\hline 21 & 30 & 20 & 30 & 28 & 25 \\
\hline 22 & 32 & 24 & 36 & 37 & 28 \\
\hline 23 & 32 & 20 & 30 & 32 & 25 \\
\hline 24 & 32 & 22 & 38 & 35 & 25 \\
\hline 25 & 27 & 22 & 36 & 35 & 23 \\
\hline 26 & 31 & 20 & 31 & 35 & 26 \\
\hline 27 & 31 & 21 & 35 & 32 & 25 \\
\hline 28 & 30 & 23 & 30 & 34 & 29 \\
\hline 29 & 28 & 20 & 33 & 33 & 28 \\
\hline 30 & 32 & 20 & 32 & 33 & 21 \\
\hline 31 & 31 & 21 & 30 & 32 & 20 \\
\hline 32 & 30 & 22 & 31 & 32 & 25 \\
\hline 33 & 30 & 19 & 30 & 36 & 28 \\
\hline 34 & 27 & 19 & 32 & 35 & 25 \\
\hline 35 & 28 & 20 & 31 & 32 & 25 \\
\hline
\end{tabular}

the general population. It is noted with interest that almost all the isolates were C. albicans, except one single isolate, which was identified as C. famata. Most of the studies done all over the world have found C. albicans as the predominant pathogen followed by non-albicans Candida species, including C. glabrata, C. parapsilosis, and C. tropicalis. ${ }^{3,9,10}$

In a previous study done in 1994 in Sri Lanka, C. albicans was also found to be the most common pathogen (76\%). ${ }^{11}$ Similarly, in the 2010 study, C. albicans was the predominant organism $(75 \%) .{ }^{8}$ In the current study, all the isolates were susceptible to all tested antifungals. In the 2010 study, majority of C. albicans strains had shown susceptibility to fluconazole, itraconazole, clotrimazole, and nystatin. ${ }^{8}$ However, only three strains in that study were resistant to econazole, and another two strains were intermediately susceptible to miconazole. As reported by
Perera and Clayton in 1994, C. albicans isolates showed resistance to miconazole and econazole. ${ }^{11}$ The studies done over the decades in Sri Lanka reflect that antifungal resistance is not a major burden in the management of VVC. Moreover, C. albicans remains the predominant pathogen in VVC to date.

Vaginal discharge and itching are quite common symptoms among these patients, but the symptoms are insufficient to diagnose VVC in the absence of laboratory confirmation. In our study, white and viscous discharges were two main significant clinical symptoms associated with VVC. A common complaint regarding VVC is that the symptoms exacerbate with menstruation. An elevated level of estrogen has been associated with VVC. ${ }^{12}$ However, in this study we found that the patients claimed that VVC symptoms were significantly higher following menstruation. Further conclusions cannot be made with 
regard to the association of VVC with menstruation in this study as data were not collected to determine the duration following menstruation when the symptoms increased. This is one limitation of this study.

Several conditions, such as the use of antibiotics, diabetes, and pregnancy, are known risk factors for VVC. ${ }^{3,13,14}$ In our study, although we have studied several factors, only diabetes mellitus and the use of oral contraceptives could be identified as risk factors. Therefore, it is important to investigate other possible causes predisposing to VVC among these patients. It is suggested that genetic predisposition such as mutations and gene polymorphisms associated with innate immunity has a role in VVC. ${ }^{1}$

\section{CONCLUSION}

Candida albicans was the predominant organism causing VVC in this study done in a particular setting in Sri Lanka. This is a positive finding for the gynecologists treating these patients because strains of C. albicans show a low frequency of resistance to azoles; therefore, the management of these patients can be achieved successfully.

\section{ACKNOWLEDGMENT}

Authors would like to thank the medical and nursing staff at the Obstetrics and Gynecology Professorial Unit, Colombo South Teaching Hospital, Sri Lanka, for their assistance.

\section{REFERENCES}

1. Jaeger M, Plantinga TS, Joosten LA, Kullberg BJ, Netea MG. Genetic basis for recurrent vulvo-vaginal candidiasis. Curr Infect Dis Rep 2013 Apr;15(2):136-142.

2. Mohammadi R, Mirhendi H, Rezaei-Matehkolaei A, Ghahri M, Shidfar MR, Jalalizand N, Makimura K. Molecular identification and distribution profile of Candida species isolated from Iranian patients. J Med Mycol 2013 Aug; 51(6):657-663.

3. Ogouyèmi-Hounto $A$, Adisso $S$, Djamal J, Sanni $R$, Amangbegnon R, Biokou-Bankole B, Kinde Gazard D,
Massougbodji A. Place of vulvovaginal candidiasis in the lower genital tract infections and associated risk factors among women in Benin. J Mycol Med 2014 Jun;24(2):100-105.

4. Iavazzo C, Gkegkes ID, Zarkada IM, Falagas ME. Boric acid for recurrent vulvovaginal candidiasis: the clinical evidence. J Womens Health (Larchmt) 2011 Aug;20(8):1245-1255.

5. Gunther LS, Martins HP, GimenesF, de Abreu AL, ConsolaroME, Svidzinski TI. Prevalence of Candida albicans and non-albicans isolates from vaginal secretions: Comparative evaluation of colonization, vaginal candidiasis and recurrent vaginal candidiasis in diabetic and non-diabetic women. Sao Paulo Med J 2014;132(2):116-120.

6. Pfaller MA, Diekema DJ. Rare and emerging opportunistic fungal pathogens concern for resistance beyond C. albicans and Aspergillus fumigatus. J Clin Microbiol 2004 Oct;42(10): 4419-4431.

7. NCCLS. Method for antifungal disk diffusion susceptibility testing of yeast. Approved Guidelines Document M 44 A. 2004;24(15).

8. Jayasekera, PI, Perera PD, Samarakoon S. Project on Vulvovaginal Candidiasis (VVC) among female patients attending the Central STD clinic Colombo, for the first time. Oral presentation at the Annual Scientific Sessions of the Sri Lanka College of Microbiologists, 2010.

9. Mohanty S, Xess I, Hasan F, Kapil A, Mittal S, Tolosa JE. Prevalence \& susceptibility to fluconazole of Candida species causing vulvovaginitis. Indian J Med Res 2007 Sep;126(3): 216-219.

10. Diba K, Namaki A, Ayatolahi H, Hanifian H. Rapid identification of drug resistant Candida species causing recurrent vulvovaginal candidiasis. Med Mycol J 2012; 53(3):193-198.

11. Perera J, Clayton Y. Incidence, species distribution and antifungal sensitivity pattern of vaginal yeasts in Sri Lankan women. Mycoses 1994 Sep-Oct;37(9-10):357-360.

12. Fischer G. Chronic vulvovaginal candidiasis: What we know and what we have yet to learn. Australas J Dermatol 2012 Nov; 53(4):247-254.

13. Donders GG, Bellen G, Mendling W. Management of recurrent vulvo-vaginal candidosis as a chronic illness. Gynecol Obstet Invest 2010;70(4):306-321.

14. Donders GG, Mertens I, Bellen G, Pelckmans S. Selfelimination of risk factors for recurrent vaginal candidosis. Mycoses 2011Jan; 54(1):39-45. 